\title{
A NOVEL PLASTICITY-DAMAGE CONSTITUTIVE MODEL FOR UNIDIRECTIONAL LONG FIBRE REINFORCED POLYMER
}

\author{
I.R. Cózar ${ }^{1 *}$, P. Maimi ${ }^{1}$, F. Otero ${ }^{2}$, E.V. González ${ }^{1}$, P. Camanho ${ }^{3}$, S. Miot ${ }^{4}$, A. Turon ${ }^{1}$ \\ ${ }^{1}$ AMADE, Polytechnic School, Universitat de Girona, 17003 Girona, Spain \\ ${ }^{2}$ CIMNE, Universitat de Politècnica de Catalunya, 08034 Barcelona, Spain \\ ${ }^{3}$ DEMec, Faculdade de Engenharia, Universidade do Porto, 4200-465 Porto, Portugal \\ ${ }^{4}$ IRT Saint Exupéry, 31405 Toulouse, France \\ * corresponding author: ivan.ruiz@udg.edu
}

The increasing use of advanced composite materials in complex structures requires extensive experimental test campaigns to understand their mechanical response. To reduce the size of the test campaigns, efficient and reliable numerical tools are required. A new constitutive model have been developed to allow for more accurate and robust description of the mechanical behaviour of composite materials.

The constitutive model allows to predict the inelastic deformation and fracture of a transversely isotropic unidirectional composite material at the meso-scale level within the framework of the infinitesimal strain theory. In the directions governed by the polymer, the model initially accounts for an elastic behaviour until the onset of plasticity is reached. Then, plasticity is taken into account until the onset of damage. In the fibre direction, the model describes the elastic response until the onset of damage and no plasticity is considered. When a crack nucleates, it propagates without plasticity in any direction (i.e. polymer and fibre directions).

In this study, a yield function combined with non-associated flow rule is proposed. It allows for the volumetric plastic strains to be imposed. The shape of the yielding and damage surfaces can be modified as a function of two and six parameters, respectively. The damage model is based on the model developed by Quintanas et al. [1], within the framework of the crack band model of Bažant et al. [2] to ensure mesh objectivity and, thus, the proper energy dissipation when the crack propagates. Four damage variables are defined to capture the fibre breakage and the fibre kinking, and the degradation related to the mode-I and mode-II matrix cracking.

The constitutive model has been implemented in a user material subroutine to be used with Abaqus/Explicit solver (VUMAT). The constitutive model has been used for the simulation of different tests on polymer-based composite structures. A good correlation of the numerical results with experimental data was observed.

This work has received funding from the VITAL private project and Clean Sky 2 Joint Undertaking under the European Union's Horizon 2020 research and innovation programme under Grant Agreement Number 864723.

\section{References}

[1] A. Quintanas-Corominas, P. Maimí, E. Casoni, A. Turon, J. A. Mayugo, G. Guillamet, M. Vázquez, A 3d transversally isotropic constitutive model for advanced composites implemented in a high performance computing code, European Journal of MechanicsA/Solids 71 (2018) 278-291.

[2] Z. P. Bažant, B. H. Oh, Crack band theory for fracture of concrete, Matériaux et construction 16 (3) (1983) 155-177. 